\title{
A Joint Optimization Scheme of Resource Allocation in Downlink NOMA with Statistical Channel State Information
}

Lei Xu ( $\square$ ahu_xulei@foxmail.com)

School of Electronics and Information Engineering, Anhui University https://orcid.org/0000-0001-70269310

Jing Yi Yao

School of Electronics and Information Engineering,Anhui University

Jing Cai

School of Electronics and Information Engineering, Anhui University

Yu Hong Fang

School of Electronics and Information Engineering, Anhui University

Hui Xiao Li

School of Electronics and Information Engineering, Anhui University

\section{Research}

Keywords: Non-orthogonal multiple access, statistical channel state information, user grouping, power allocation, throughput

Posted Date: August 14th, 2020

DOl: https://doi.org/10.21203/rs.3.rs-57297/v1

License: (c) (1) This work is licensed under a Creative Commons Attribution 4.0 International License. Read Full License 


\title{
RESEARCH
}

\section{A joint optimization scheme of resource allocation in downlink NOMA with statistical channel state Information}

\author{
Lei $\mathrm{Xu}^{*}$, YiJing Yao, Jing Cai, Hongyu Fang and Xiaohui Li
}

* Correspondence:
xulei@ahu.edu.cn
School of Electronics and
Information Engineering, Anhui
University, Jiulong Road, Hefei,
China
Full list of author information is
available at the end of the article

${ }^{*}$ Correspondence:

xulei@ahu.edu.c

Information Engineering, Anhui

China

available at the end of the article

\begin{abstract}
In a real communication scenario, it is very difficult to obtain the real-time Channel State Information(CSI) accurately, so the communication systems with statistical CSI have been researched. In order to maximize the throughput of the downlink Non-Orthogonal Multiple Access (NOMA) system with statistical CSI, the formula of system throughput is derived at first. Then, according to the combinatorial characteristics of the original optimization problem, it is divided into two subproblems, that is user grouping and power allocation. At last, a joint optimization scheme is proposed. In which, Genetic algorithm is introduced to solve the subproblem of power allocation, and Hungarian algorithm is introduced to solve the subproblem of user grouping. By comparing the ergodic date rate of NOMA users with statistical CSI and perfect CSI, the effectiveness of the statistical CSI sorting is verified. Compared with the Orthogonal Multiple Access (OMA) scheme, the NOMA scheme with the fixed user grouping scheme and the random user grouping scheme, the proposed scheme can effectively improve the system throughput.
\end{abstract}

Keywords: Non-orthogonal multiple access; statistical channel state information; user grouping; power allocation; throughput

\section{Introduction}

Non-Orthogonal Multiple Access (NOMA) technology has recently attracted tremendous attention due to its simple design and superior spectrum efficiency, which is recognized as a promising multiple access scheme in the next generation mobile communication networks[1]. By splitting multiple users via different transmission power, Superposition Coding (SC), adopted by NOMA, introduces interference information, so Successive Interference Cancellation (SIC) is required at receiver side to realize multi-user detection[2]. Many of the existing works about NOMA have assumed the perfect Channel State Information (CSI) at transmitter side, which are nearly impractical for many communication scenarios. Therefore, it is of great significance to investigate the resource optimization of downlink NOMA system with statistical CSI.

Recently, some literature has investigated the resource optimization problem in NOMA system with statistic CSI. In [3], the performance of two NOMA system with partial CSI has been evaluated, and the research results show that statistical CSI based on second order statistics is always better than the incomplete CSI 
based on feedback, and the system performance with statistical CSI is similar to that with perfect CSI, in the case of low Signal to Interference plus Noise Ratio (SINR). The power allocation scheme of the NOMA system is investigated[4], a sub-optimal power allocation algorithm is proposed. In which, Genetic Algorithms (GA) dynamically allocates users in a group, but the design of user grouping scheme is neglected. In [5], a dynamic power allocation scheme and a user grouping algorithm are proposed, users are divided into to two sets according to their statistic CSI, and the users, in different sets and with the same sort number, are matched into one group. This grouping method is simple to implement, but it cannot guarantee the overall performance of the system. And this literature proves that the performance of the NOMA system is proportional to the difference in statistical characteristics between users within the same group. In [6], a low complexity power allocation sub-optimal solution and a user scheduling scheme are proposed, based on a hierarchical clustering algorithm are proposed. In which, energy efficiency is used as the evaluation index, and the near-optimal performance can be achieved.

The aforementioned literature all considers user grouping and power allocation separately. But in fact, user grouping and power allocation are intertwined with each other[7]. Since multiple users are admitted simultaneously, when the number of users is large, the complexity of the SIC receiver in the NOMA system is very high. As a result, it may not be realistic to ask all users in the system to perform NOMA jointly, a promising alternative is to construct a hybrid multiple access system. In which, NOMA is combined with Conventional Multiple Access (OMA)[8]. The users in the system are divided into multiple groups, where NOMA is implemented within each group and different groups are allocated with orthogonal resources. Obviously, the performance of the NOMA system is very dependent on which users are grouped together and the power allocation between users [9]. Therefore, effective user grouping and power allocation schemes can provide feasibility for improving the performance of the downlink NOMA system with statistical CSI.

From what has been discussed above, this paper derives the system throughput formula, and proposes a joint power allocation and user grouping resource allocation optimization scheme, combined the advantages of GA and Hungarian Algorithm (HA) in the field of resource optimization. Firstly, according to the characteristics of the original problem, the problem is divided into two subproblems, that is power allocation and user grouping. Secondly, under arbitrary and fixed user grouping scheme, a power allocation algorithm is proposed to maximize the intra-group throughput, combined the advantages of GA in solving non-convex problems. Finally, the user grouping problem is equivalent to a matching problem, a user grouping scheme, which can maximize the system throughput, is obtained, combined the low-complexity characteristics of the HA to solve the matching problem. The symbols used in this paper are defined as follows: $E(\cdot)$ represents the mean operator, $f(\cdot)$ and $F(\cdot)$ represent the Probability Density Function (PDF) and the Cumulative Distribution Function (CDF), respectively. and $U$ represents the uniform distribution. 


\section{System model}

In this paper, a single-cell downlink multi-user system is considered, which adopts the hybrid multiple access scheme. The Base Station (BS) is located in the center of the cell. $N$ users are randomly distributed in the cell, and both the users and the BS are equipped with a single antenna. The users are divided into $L$ groups, and each group contains $K_{l}$ users, which satisfies

$$
\sum_{l=1}^{L} K_{l}=N
$$

NOMA is implemented within each group and different groups are allocated with orthogonal resources to eliminate inter-group interference, and some intra-group interference can be eliminated by SIC.

Without loss of generality, this paper adopts the widely used Rayleigh fading channel for communication, that is affected by the joint effect of large-scale fading and small-scale fading. The channel model can be modeled as

$$
h_{n}=\frac{g_{n}}{\sqrt{1+d_{n}^{\alpha}}}
$$

where $g_{n}$ denotes the small-scale fading coefficient, which subjects to $g_{n} \sim$ $\mathcal{C N}(0,1) . d_{n}$ denotes the distance between user $n$ and the BS, and $\alpha$ denotes the average path loss factor. The channel coefficient $h_{n}$ subjects to the Rayleigh distribution, and its PDF can be expressed as

$$
f\left(x ; \Omega_{n}\right)=\frac{2 x}{\Omega_{n}} e^{-\frac{x^{2}}{\Omega_{n}}}, x \geq 0
$$

where $\Omega_{n}$ denotes the large-scale fading coefficient between user $n$ and BS, expressed as $\Omega_{n}=E\left(\left|h_{n}\right|^{2}\right)$. Assume that the BS only has statistical CSI related to each user, i.e., the BS knows the value of $\Omega$ for all users.

In order to simplify the derivation of the problem without loss of generality, this paper analyzes the $l$-th group with $K_{l}$ users, so the signal received by the $k$-th user in the $l$-th group can be represented as

$$
y_{l, k}=h_{l, k} \sqrt{a_{l, k} P} x_{l, k}+h_{l, k} \sum_{i \neq k} \sqrt{a_{l, i} P} x_{l, i}+z_{l, k}
$$

where $P$ denotes the transmit power allocated by the BS to each group, this paper assumes that the transmission power BS allocates to each group is equal. $h_{l, k}$ denotes the instantaneous channel related to the $k$-th user in the $l$-th group, $x_{l, k}$ denotes the transmitted message intended for the $k$-th user in the $l$-th group, $a_{l, k}$ denotes the intra-group power allocation factor allocated to the $k$-th user in the $l$-th group, $z_{l, k}$ denotes independent and identically distributed additive white Gaussian noise, which subjects to $z_{l, k} \sim \mathcal{C N}\left(0, \sigma_{2}\right)$. Without loss of generality, the paper assumes that the users are sorted in the ascending order of statistic CSI, i.e., 
$\Omega_{l, 1}<\Omega_{l, 2}<\cdots<\Omega_{l, K}$. According to the principle of NOMA, it can be concluded that the power allocation factor is satisfies $a_{l, 1}>a_{l, 2}>\cdots>a_{l, K}$. The second term in (4) is caused by the user's intra-group interference, which can be partially eliminated by the SIC receiver. According to the optimal SIC decoding order under the statistical CSI proposed in [10], the decoding order of the SIC within the group is $\left(1,2, \cdots, K_{l}\right)$, which is the increasing order of channel gain. The user detection is shown in Fig.1.

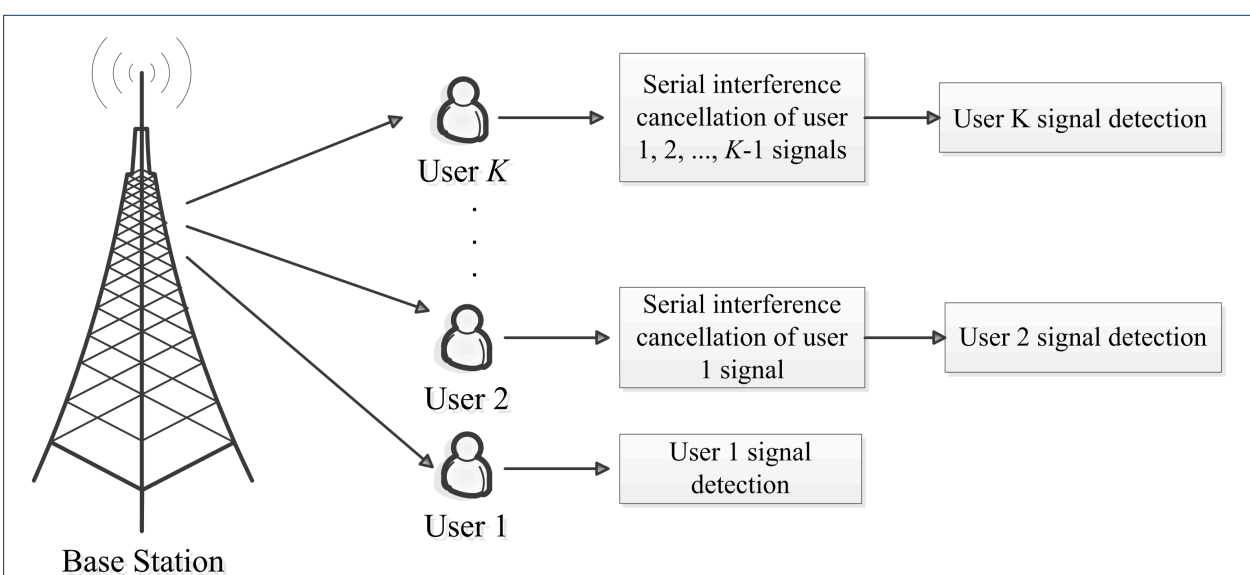

Figure 1 User detection model

Ideally, in the case of $j<k<m$, the $k$-th user will detect the message of $j$ th user, and then removes the message from its received in a successive manner. The message of $m$-th user will be treated as noise at the $k$-th user. Therefore, the instantaneous date rate of the $k$-th user in the $l$-th group is represented as

$$
R_{l, k}=\log _{2}\left(1+\frac{\rho\left|h_{l, k}\right|^{2} a_{l, k}}{\rho\left|h_{l, k}\right|^{2} \sum_{m=k+1}^{K_{1}} a_{l, m}+1}\right)
$$

where $\rho=P / \sigma^{2}$ denotes the intra-group SINR. Note that the date rate of the $K_{l}$-th user is $R_{l, K_{l}}=\log _{2}\left(1+\rho\left|h_{l, K_{l}}\right|^{2} a_{l, K_{l}}\right)$. Let $R_{l, k \rightarrow l, j}$ denotes the instantaneous date rate of the $k$-th user detects the $j$-th user's message in $l$-th group, which can be represented as

$$
R_{l, k \rightarrow l, j}=\log _{2}\left(1+\frac{\rho\left|h_{l, k}\right|^{2} a_{l, j}}{\rho\left|h_{l, k}\right|^{2} \sum_{i=j+1}^{K_{1}} a_{l, i}+1}\right)
$$




\section{Performance analysis}

Due to the analysis of outage probability and ergodic data rate require the density function of channel, this paper adopts the widely used Rayleigh fading channel for communication, therefore, the PDF and CDF of unsorted channel gain are as follows

$$
\begin{aligned}
& f_{\left|\tilde{h}_{n}\right|^{2}}\left(x ; \Omega_{n}\right)=\frac{1}{\Omega_{n}} e^{-\frac{x}{\Omega_{n}}}, x \geq 0 \\
& F_{\left|\tilde{h}_{n}\right|^{2}}\left(x ; \Omega_{n}\right)=1-e^{-\frac{x}{\Omega_{n}}}, x \geq 0
\end{aligned}
$$

Since the users are sorted in the ascending order of statistic CSI, the corresponding PDF and CDF of the ordered channel gain can be written as[11]

$$
\begin{aligned}
& f_{\left|h_{n}\right|^{2}}\left(x ; \Omega_{n}\right)=\beta_{N, n} \sum_{t=0}^{N-n}(-1)^{t}\left(\begin{array}{c}
N-n \\
t
\end{array}\right) f_{\left|\tilde{h}_{n}\right|^{2}}\left(x ; \Omega_{n}\right)\left[F_{\left|\tilde{h}_{n}\right|^{2}}\left(x ; \Omega_{n}\right)\right]^{n+t-1} \\
& F_{\left|h_{n}\right|^{2}}\left(x ; \Omega_{n}\right)=\beta_{N, n} \sum_{t=0}^{N-n} \frac{(-1)^{t}}{n+t}\left(\begin{array}{c}
N-n \\
t
\end{array}\right)\left[F_{\left|\tilde{h}_{n}\right|^{2}}\left(x ; \Omega_{n}\right)\right]^{n+t}
\end{aligned}
$$

where $\beta_{N, n}=\frac{N !}{(N-n)(n-1) !}$.

\subsection{Ergodic date rate}

In order to compare the impact of statistical CSI and perfect CSI on the performance of the NOMA system, based on (5) and (9), the ergodic data rate expression of user $\mathrm{k}$ is derived, and Monte Carlo method is adopted to solve it.

$$
\begin{aligned}
R_{k}^{\text {NOMA }} & =\int_{0}^{+\infty} R_{k} f_{|h|^{2}}\left(x ; \Omega_{k}\right) d x \\
& =E_{x \sim f_{\left|h_{n}\right|^{2}}\left(x ; \Omega_{n}\right)}\left[\log _{2}\left(1+\frac{a_{k} x}{\rho\left(x \sum_{i=k+1}^{K} a_{i}+1\right)}\right)\right]
\end{aligned}
$$

\subsection{Outage probability}

For the sake of guarantee the SIC decoding in section 2 is performed correctly, $R_{l, k \rightarrow l, j} \geq R_{l, j}^{t h}$ must be satisfied, where $R_{l, j}^{t h}$ denotes the target date rate of the $j$-th user in the $l$-th group. When the instantaneous date rate cannot reach the target date rate, outage occurs. Therefore, the outage probability at the k-th user can be expressed as follows [12]

$$
P_{l, k}^{N O M A}=1-\operatorname{Pr}\left(\bigcap_{j=1}^{k}\left(R_{l, k \rightarrow l, j} \geq R_{l, j}^{t h}\right)\right)
$$

where $\left(R_{l, k \rightarrow l, j} \geq R_{l, j}^{t h}\right.$ can be expressed as follows

$$
\left|h_{l, k}\right|^{2} \geq \frac{\varepsilon_{l, j}}{\rho\left(a_{l, j}-\varepsilon_{l, j} \sum_{i=j+1}^{K} a_{l, i}\right)}
$$


where $\varepsilon_{l, j}=2^{R_{l, j}^{t h}}-1$. Note that (13) is obtained by assuming the the following condition holds

$$
a_{l, k}>\varepsilon_{l, j} \sum_{i=k+1}^{K} a_{l, i}
$$

Otherwise, the outage probability is always 1 , furthermore, the outage probability can be written as follows

$$
P_{l, k}^{N O M A}=\operatorname{Pr}\left(\left|h_{l, k}\right|^{2}<\max \left\{\frac{\varepsilon_{l, j}}{\rho\left(a_{l, j}-\varepsilon_{l, j} \sum_{i=j+1}^{K_{l}} a_{l, i}\right)}\right\}\right)
$$

Let

$$
\phi_{l, k}=\max \left\{\frac{\varepsilon_{l, j}}{\rho\left(a_{l, j}-\varepsilon_{l, j} \sum_{i=j+1}^{K_{l}} a_{l, i}\right)}\right\}
$$

Based on (10) and (15), the user outage probability expression of the NOMA system with statistical CSI can be obtained as

$$
P_{l . k}^{N O M A}=\beta_{K_{l}, k} \sum_{t=0}^{K_{l}-k} \frac{(-1)^{t}}{k+t}\left(\begin{array}{c}
K_{l}-k \\
t
\end{array}\right)\left[1-e^{-\frac{\phi_{l, k}}{\Omega_{l, k}}}\right]^{k+t}
$$

\subsection{Throughput}

In general, there are mainly two category of methods for optimizing the performance in communication systems. One is to maximize the achievable sum date rate, and the other one is to ensure the fairness of users[13]. On the one hand, to maximize the sum date rate, the BS tends to allocate more power to users with high channel gains, which may cause users with low channel gains to be outage. On the other hand, the fairness among the users may result in the performance loss of the achievable sum date rate. In order to achieve a trade-off between achievable sum date rate and user fairness, this paper is consider to maximize the achievable sum date rate while ensuring the minimum rate requirement of each user, therefore, throughput is adopted to characterize rate performance. Throughput combines rate performance and fairness, which is defined as the sum of target date rate of each user multiplied by its successful transmission probability [14], expressed as follows

$$
T^{N O M A}=\sum_{l=1}^{L} \sum_{k=1}^{K_{l}} R_{l, k}^{t h}\left(1-P_{l, k}^{N O M A}\right)
$$


the target date rate represents the rate performance, and the probability of successful transmission ensures the fairness of users. In order to verify the superiority of the NOMA system performance, Time Division Multiple Access (TDMA) system was selected for comparison. Assuming that the system is of equal time slots, and the transmission power allocated to each time slot is equal, the instantaneous date rate achieved is given by

$$
R_{n}^{O M A}=\frac{1}{N} \log _{2}\left(1+\frac{P_{\text {total }}\left|h_{n}\right|^{2}}{\sigma^{2}}\right)
$$

where $P_{\text {total }}$ denotes the total transmit power of the BS. Similar to the derivation process of formula (17) and (18), the corresponding outage probability of user $n$ and throughput can be calculated respectively as

$$
\begin{aligned}
P_{n}^{O M A} & =1-e^{-\frac{\left(2^{N R_{n}^{t h}-1}\right) \sigma^{2}}{P_{t o t a l} \Omega_{n}}} \\
T^{O M A} & =\sum_{n=1}^{N} R_{n}^{t h}\left(1-P_{n}^{O M A}\right)
\end{aligned}
$$

where $R_{n}^{t h}$ denotes the target date rate of user $n$.

\section{Resource allocation optimization scheme}

As analyzed in Section 3.3, in order to realize the trade-off between system achievable sum date rate and user fairness, throughput is adopted to characterize rate performance in this paper. Therefore, the problem of maximizing throughput can be represented as follows

$$
\begin{array}{ll}
\max & \sum_{l=1}^{L} \sum_{k=1}^{K_{l}} R_{l, k}^{t h}\left(1-P_{l, k}^{N O M A}\right) \\
\text { s.t. } & C_{1}: \sum_{l=1}^{L} P \leq P_{\text {total }}, P>0 \\
& C_{2}: \sum_{k=1}^{K_{l}} a_{l, k}=1, a_{l, k}>0 \\
& C_{3}: a_{l, 1}>a_{l, 2}>\cdots>a_{l, K_{l}} \\
& C_{4}: a_{l, k}>\varepsilon \sum_{i=k+1}^{K_{l}} a_{l, i}
\end{array}
$$

where the constraint condition $C_{1}$ is the total transmit power constraint, and the power allocated to each group should be non-negative. The constraint condition $C_{2}$ is the power allocation factor constraint for each group. The constraint conditions $C_{3}$ and $C_{4}$ represent the NOMA principle constraints.

The solution to maximize system throughput in (22) is not only affected by the power allocation factor, but also depends on which users can be allocated to the same group. In many of the existing works, users are grouped directly according to their channel condition, and then power is allocated to users within each group. 
However, it ignores the inherent relationship between power allocation and user grouping. Therefore, this paper proposes a joint resource allocation optimization algorithm. The solution to original problems can be divided into two stages. In the first stage, under arbitrary and fixed user grouping scheme, an intra-group power allocation scheme is proposed to maximize intra-group throughput. In the second stage, combined with the intra-group power allocation scheme, a user grouping scheme is given for the sake of maximizing system throughput.

\subsection{Power allocation}

Under arbitrary and fixed user grouping scheme, this paper assumes transmission power allocated to each group is equal. Therefore, the problem of maximizing system throughput in (22) can be divided into multiple maximizing intra-group throughput problems.

$$
\begin{aligned}
\max & \sum_{k=1}^{K_{l}} R_{k}^{t h}\left(1-P_{k}^{N O M A}\right) \\
& C_{1}: \sum_{k=1}^{K_{l}} a_{k}=1, a_{k}>0 \\
& C_{2}: a_{1}>a_{2}>\cdots>a_{K_{l}} \\
& C_{3}: a_{k}>\varepsilon \sum_{i=k+1}^{K_{l}} a_{i}
\end{aligned}
$$

Due to the objective function is non-convex, exhaustive search for the optimal solution results in heavy complexity, which is hard to accomplish in practice. Therefore, GA is adopted for power allocation.

GA is based on natural selection and genetic theory, which combines the survival of the fittest in the process of biological evolution with the random information exchange mechanism of chromosomes within the population. GA abandons the traditional search method, simulates the biological evolution process in nature, and uses artificial evolution to search the target space randomly. It regards the possible solution in the problem domain as an individual or chromosome of the group, and encodes each individual into a symbol string form, simulating the biological evolution process of Darwin's genetic selection and natural elimination, and iterative operations selection, crossover and mutation based on genetics are performed on the population. According to the predetermined target fitness function, each individual is evaluated, according to the evolutionary rules of survival of the fittest and survival of the fittest, a better group is continuously obtained. At the same time, the global parallel searcher is used to search for the optimal individual in the optimization group to find the optimal solution that meets the requirements[15].

In traditional GA, the parameters of the three operators of selection, crossover and mutation are fixed, which limit and restrict the performance of GA. Fixed parameters may lead to the destruction of the optimal individual, and the evolutionary process does not converge. In the later stage of evolution, the diversity of individuals in the population is greatly reduced, and the fitness value is close, causing the algorithm to approach a random search state. 
In order to alleviate the above problems, this paper considers the dynamic determination of mutation probability according to the fitness value of the individual, it can not only prevent good genes from being destroyed due to mutation, but also introduce new genes into the population when it falls into a local optimal solution. Improve the crossover method, after selecting the male parent and the female parent, perform multiple crossovers according to single-point crossover, multi-point crossover and uniform crossover, and select the best two individuals from the offspring individuals to join the new population. The improved algorithm is described as follows

a) Initialize population number $S$ and the maximum generation $G_{\max }$, randomly generate $S$ individuals represented by a binary string of length $N_{b s}$ placed in the initial population $Q(0)$.

b) Regard (22) as the fitness function, and compute the fitness value $f$ of each individual.

c) According to the fitness value $f$ of the individual, the selection probability $P_{s}^{i}=$

$f_{i} / \sum_{k=1}^{K_{l}} f_{k}$, crossover probability $P_{c}$ and mutation probability $P_{m}^{i}=\left(1-P_{s}^{i}\right) P_{m}$ of each individual is computed, where $P_{c}$ and $P_{m}$ represent the crossover probability and mutation probability initially set by the algorithm.

d) The individuals in the population $Q(G)$ are selected and mutated firstly, then randomly select two individuals, crossover them multiple times according to the crossover probability $P_{c}$, finally select the best two individuals from the offspring individuals to obtain the next population $Q(G+1)$.

e) If $G<G_{\max }$, go to $b$ ); else, stop the algorithm and get the individual with greatest fitness as the solution.

\subsection{User grouping}

It should be noted that in 3GPP LTE Advanced, two users are selected for performing NOMA [16], and the number of users in each group $K_{l}$ cannot too large take consider of complexity of SIC. Therefore, the user grouping scheme for the case of $K_{l}=2$ is investigated. The user grouping problem can be modeled as

$$
\arg \max \sum T_{i, j}^{N O M A}
$$

where $T_{i, j}^{N O M A}$ denotes the maximum intra-group throughput when user $i$ and user $j$ are allocated to the same group.

Since channel differences have a direct impact on user grouping, users are divided into two sets based on the statistical CSI, denoted as $V_{1}$ and $V_{2}$. Specific steps are as follows

a) Detect the $\Omega$ of users in the cell, and users are sorted in the ascending order of $\Omega$. 
b) Take user $n\left(n=f_{\text {floor }}(N / 2)\right)$ as the boundary and divide users into two sets $V_{1}$ and $V_{2}$, where $f_{\text {floor }}(\cdot)$ means rounding to the left.

If $N$ is an even number, the numbers of users in $V_{1}$ and $V_{2}$ are equal. If $N$ is an odd number, the first user in $V_{2}$ is taken out as a user group, then a user is selected from set $V_{1}$ and set $V_{2}$ respectively to form a two-user group. After the above processing, enlarge channel differences between users within the same group can improve NOMA system performance gain. However, arbitrary matching of users in the two sets cannot guarantee the system throughput performance. Considering the impact of power allocation on user grouping, the user grouping problem can be transformed into a one-to-one matching problem between user sets V1 and V2 for sake of maximizing scheme throughput. Taking advantage of the low complexity feature of the HA to solve the matching problem, this paper introduces the HA to solve (24).

The HA is one of the classic algorithms for bipartite graph matching in graph theory. Its application background is to solve the problem of two-dimensional task allocation. HA involves two concepts, namely bipartite graph and augmenting-path. Bipartite graph is a special model in graph theory. Let $\mathbf{A}=(\mathbf{B}, \mathbf{C})$ be an undirected graph. If the vertex $\mathbf{B}$ can be divided into two disjoint subsets $\left(\mathbf{B}_{1}, \mathbf{B}_{2}\right)$, and the two vertices $i$ and $j$ associated with each edge $(i, j)$ in the graph belong to these two different vertex sets, so $\mathbf{A}$ is called a bipartite graph. For augmenting-path, let $\mathbf{M}$ be the set of matched edges in the bipartite graph $\mathbf{A}$, if $\mathbf{X}$ is a path connecting two unmatched vertices in the graph $\mathbf{A}$, and edges belonging to $\mathbf{M}$ and edges not belonging to $\mathbf{M}$ appear alternately on $\mathrm{X}$, then call $\mathrm{X}$ an augmenting-path relative to $\mathrm{M}$. The basic idea of HA is to exchange the matching and non-matching edges in the augmenting-path by searching for the augmenting-path, so that there will be one more matching edge until no augmenting-path is found.

The HA to solve (24) can be equivalent to selecting $W$ elements from the $W \times$ $W\left(W=f_{\text {floor }}(N / 2)\right)$ matrix shown in $(25)$ to maximize their sum, and any of these $W$ elements are not on the same row and the same column. The selected element denotes the maximum intra-group throughput achieved when users $i$ and $j$ are allocated to the same group.

$$
\left[\begin{array}{cccc}
T_{1,1}^{N O M A} & T_{1,2}^{N O M A} & \ldots & T_{1, W}^{N O M A} \\
T_{2,1}^{N O M A} & T_{2,2}^{N O M A} & \ldots & T_{2, W}^{N O M A} \\
\vdots & \vdots & \ddots & \vdots \\
T_{W, 1}^{N O M A} & T_{W, 2}^{N O M A} & \cdots & T_{W, W}^{N O M A}
\end{array}\right]
$$

In order to prove the effectiveness of the proposed grouping method, in this paper, the GA is also used in all comparison schemes. In terms of algorithm complexity, the time complexity of the exhaustive search is $O(N !)$. The time complexity of the HA used in this paper mainly comes from the sorting process, which is $O\left(W^{2}+\right.$ $\left.W \log _{2} W\right)$, and the time complexity of the fixed matching scheme used in [5] is $O\left(W \log _{2} W\right)$. The time complexity of the proposed scheme is slightly higher than that of the fixed matching scheme, but much lower than the exhaustive search scheme, so it is still feasible even under a huge number of users. 


\section{Simulation results and analysis}

The system simulation adopts Monte Carlo method and assumes that the number of users in each group is 2, and the power allocated to each group is equal. The comparisons of ergodic data rate with perfect CSI and statistical CSI is shown in Fig. 2.

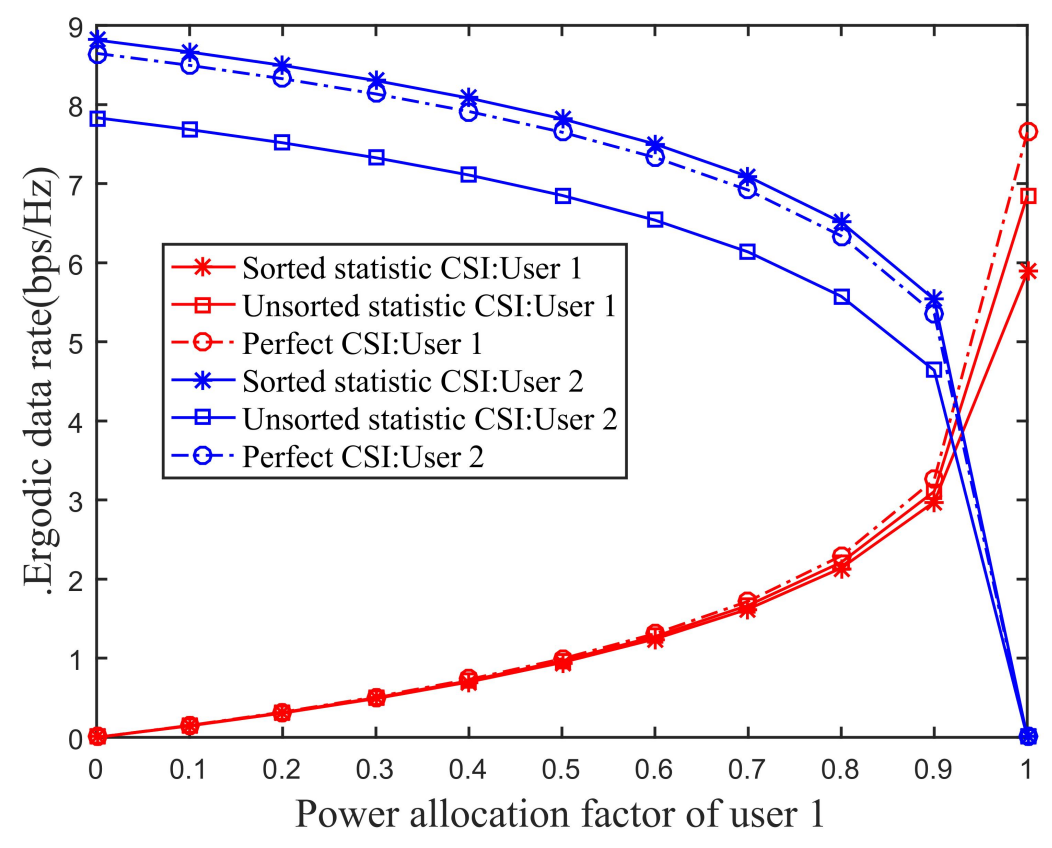

Figure 2 Comparison of ergodic date rate of NOMA users with statistical CSI and perfect CSI

As can be seen from Fig. 2, there is a certain gap between systems using statistical CSI and perfect CSI. In this paper, the users are sorted in the ascending order of statistic CSI, so the gap between the statistical CSI and the perfect CSI is reduced, and makes statistical CSI more feasible in practical applications. In Fig. 2, user 1 and user 2 represent the user with poor channel and good channel in a group respectively. When the power allocation factor allocated to user 1 is less than 0.9 , the ergodic date rate of user 2 is always much higher than that of user 1 . When the power allocated to user 1 is small, user 1 may be outage due to the constraint of minimum rate requirement, so the fairness of weak users cannot be guaranteed. However, when the power allocation factor of user 1 exceeds 0.9 , the ergodic date rate of user 2 will decrease rapidly, which will affect the overall system rate performance, this is consistent with the result analyzed in Section 3.3.

The simulation parameters in Fig. 3 are as follows: $\mathrm{SNR}=20 \mathrm{~dB}$, the statistic CSI $\Omega$ and target rateof users are generated by the system, which subject to $\Omega \sim U(0,5)$ and $R^{\text {th }} \sim U(1,5)$ respectively. In GA, the number of individuals in the population is $S=40$, length of binary string $N_{b s}=20$, the maximum generation $G_{\max }=100$, and the initially set of crossover probability and mutation probability is $P_{c}=0.7$ 


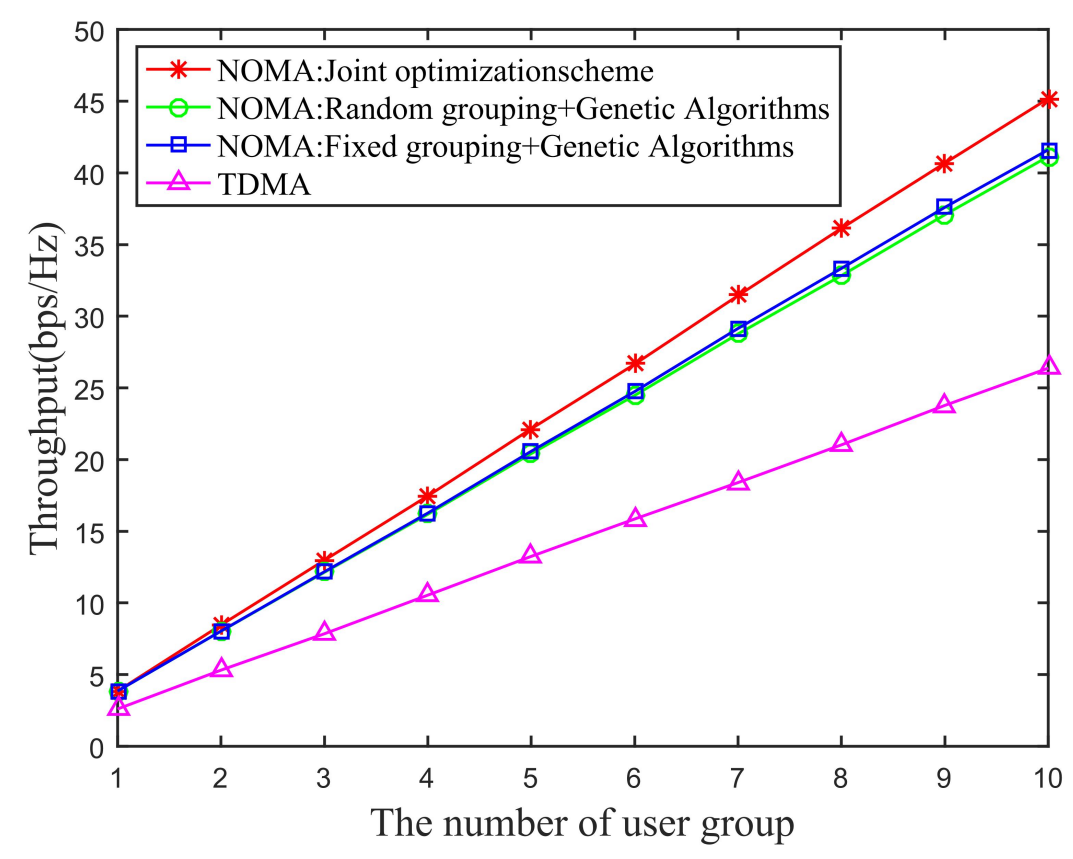

Figure 3 Trend of system throughput with the number of users

and $P_{m}=0.05$. As can be seen from Fig. 3, compared with the traditional OMA system, NOMA system can significantly improve the system throughput. For further comparison, GA is used in all schemes to allocate power to users. It can be seen that the gap between the random grouping scheme and the fixed user grouping scheme is always small, and the fixed grouping scheme proposed in [5] has achieved the largest channel difference. This demonstrates that increasing the channel difference between users can improve the system throughput, but the advantage brought by the channel difference is reduced after the user power allocation within the group. The resource allocation optimization scheme proposed in this paper is obviously better than the random grouping scheme and fixed grouping scheme in [5]. As the number of user groups served by the BS increases, the advantages of the proposed scheme are more obvious than other schemes. That is the proposed optimization scheme is more suitable for the scenario with a large number of user.

The simulation parameters of Fig. 4 are as follows: the number of users is $N=$ 12 , the statistic CSI $\Omega$, target rate $R^{t h}$ of users and the parameters of GA are consistent with Fig. 3. It can be seen from Fig. 4 that, in low SINR region, the system throughput of several schemes are similar with each other. That is because of that, the user date rate is low and outage probability is high due to the too low SINR. In high SINR region, the system allocates power to stronger users as much as possible under the constraints of NOMA principles, the system throughput of proposed grouping scheme and that of the fixed matching grouping scheme are close. However, in practical applications, the SNIR is generally between 10-20 dB. In this case, the system throughput of proposed optimization schemes are better than the 


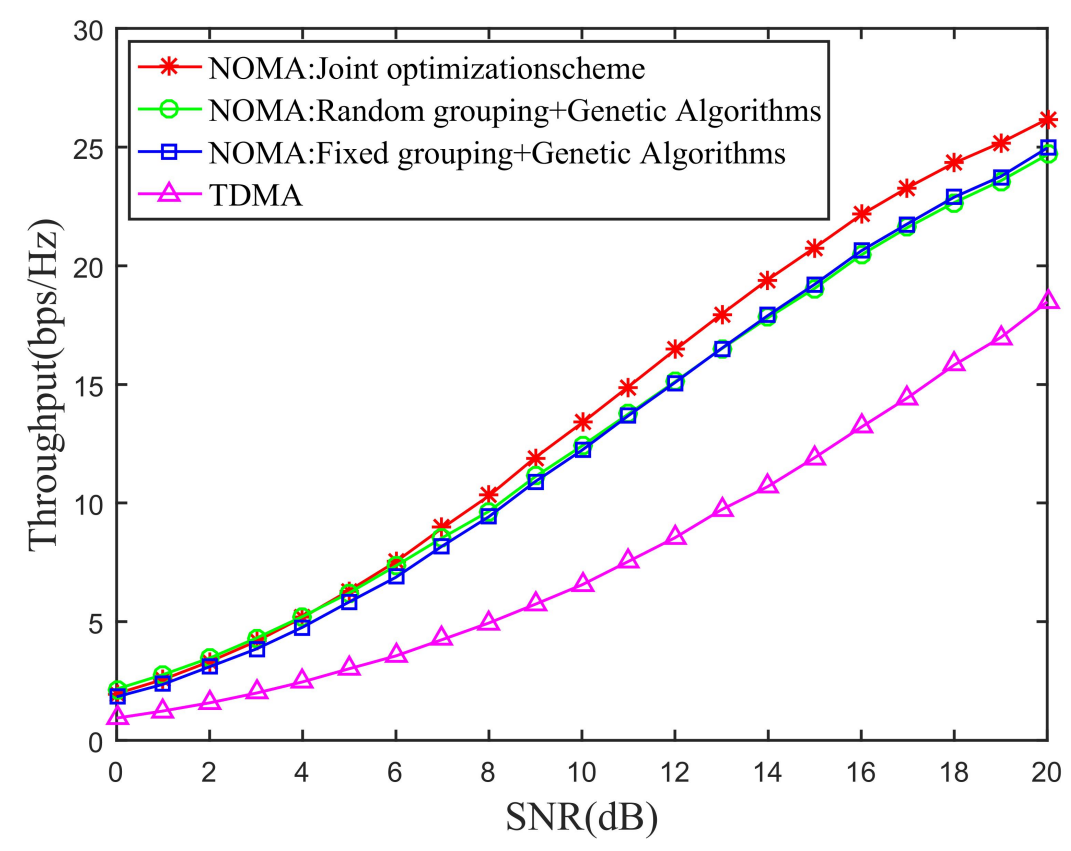

Figure 4 Trend of system throughput with the SINR

other three schemes. Compared with the TDMA scheme, random grouping scheme and the fixed grouping scheme, it can be improved by about $9 \mathrm{bps} / \mathrm{Hz}, 2.1 \mathrm{bps} / \mathrm{Hz}$ and $2 \mathrm{bps} / \mathrm{Hz}$, respectively.

\section{Conclusion}

A resource allocation optimization scheme, based on joint GA and HA, is proposed in this paper. Users are sorted in the ascending order of statistic CSI, so the performance of NOMA system with statistical CSI can further approach the NOMA system with perfect CSI. The paper derives the closed-form formula of each user's outage probability, and analyzes the outage performance and achievable sum date rate. Then throughput is adopted to characterize system performance. For the sake of maximum system throughput, GA and HA is utilized for resource allocation. Simulation results verify the effectiveness of the proposed scheme in improving the performance of system throughput.

\section{Abbreviations}

CSI: Channel State Information; NOMA: Non-Orthogonal Multiple Access; OMA: Orthogonal Multiple Access; SC: Superposition Coding; SIC: Successive Interference Cancellation; SINR: Signal to Interference plus Noise Ratio; GA: Genetic Algorithm; HA: Hungarian Algorithm; PDF: Probability Density Function; CDF: Cumulative Distribution Function; BS: Base Station; TDMA: Time Division Multiple Access.

Competing interests

The authors declare that they have no competing interests.

Funding

Specialized Research Fund for the Doctoral Program of Higher Education(No.20133401110003); Key Projects of Provincial Excellent Young Talents Fund for Universities in Anhui Province (No.2013SQRL008ZD).

Availability of data and materials

Data sharing not applicable to this article as no datasets were generated or analyzed during the current study. 
Author's contributions

LX has contributed $60 \%$ of the work. All authors read and approved the final manuscript.

References

1. Cai, Y., Qin, Z., Cui, F., Li, G.Y., McCann, J.A.: Modulation and multiple access for $5 \mathrm{~g}$ networks. IEEE Communications Surveys Tutorials 20(1), 629-646 (2018)

2. Islam, S.M.R., Zeng, M., Dobre, O.A., Kwak, K.: Resource allocation for downlink noma systems: Key techniques and open issues. IEEE Wireless Communications 25(2), 40-47 (2018)

3. Yang, Z., Ding, Z., Fan, P., Karagiannidis, G.K.: On the performance of non-orthogonal multiple access systems with partial channel information. IEEE Transactions on Communications 64(2), 654-667 (2016)

4. Wang, X., Wang, J., He, L., Song, J.: Outage analysis for downlink noma with statistical channel state information. IEEE Wireless Communications Letters 7(2), 142-145 (2018)

5. Gong, M., Yang, Z.: The application of antenna diversity to noma with statistical channel state information. IEEE Transactions on Vehicular Technology 68(4), 3755-3765 (2019)

6. Wei, Z., Ng, D.W.K., Yuan, J.: Power-efficient resource allocation for mc-noma with statistical channel state information. In: 2016 IEEE Global Communications Conference (GLOBECOM), pp. 1-7 (2016)

7. Zhu, L., Zhang, J., Xiao, Z., Cao, X., Wu, D.O.: Optimal user pairing for downlink non-orthogonal multiple access (noma). IEEE Wireless Communications Letters 8(2), 328-331 (2019)

8. Ding, Z., Fan, P., Poor, H.V.: Impact of user pairing on $5 \mathrm{~g}$ nonorthogonal multiple-access downlink transmissions. IEEE Transactions on Vehicular Technology 65(8), 6010-6023 (2016)

9. Zhang, X., Wang, J., Wang, J., Song, J.: A novel user pairing in downlink non-orthogonal multiple access. In: 2018 IEEE International Symposium on Broadband Multimedia Systems and Broadcasting (BMSB), pp. 1-5 (2018)

10. Tang, Z., Wang, J., Wang, J., Song, J.: On the achievable rate region of noma under outage probability constraints. IEEE Communications Letters 23(2), 370-373 (2019)

11. Men, J., Ge, J., Zhang, C.: Performance analysis for downlink relaying aided non-orthogonal multiple access networks with imperfect csi over nakagami- $m$ fading. IEEE Access 5, 998-1004 (2017)

12. Ding, Z., Yang, Z., Fan, P., Poor, H.V.: On the performance of non-orthogonal multiple access in $5 \mathrm{~g}$ systems with randomly deployed users. IEEE Signal Processing Letters 21(12), 1501-1505 (2014)

13. Zhu, L., Zhang, J., Xiao, Z., Cao, X., Wu, D.O., Xia, X.: Millimeter-wave noma with user grouping, power allocation and hybrid beamforming. IEEE Transactions on Wireless Communications 18(11), 5065-5079 (2019)

14. Do, D.T., Nguyen, M.S.V.: Outage probability and ergodic capacity analysis of uplink noma cellular network with and without interference from d2d pair. Physical Communication 37, 100898 (2019)

15. Lei, Y., Zhang, S.: MATLAB Genetic Algorithm Toolbox and Application. Xidian University Press, Xi'an (2014)

16. Xia, B., Wang, J., Xiao, K., Gao, Y., Yao, Y., Ma, S.: Outage performance analysis for the advanced sic receiver in wireless noma systems. IEEE Transactions on Vehicular Technology 67(7), 6711-6715 (2018) 
Figures

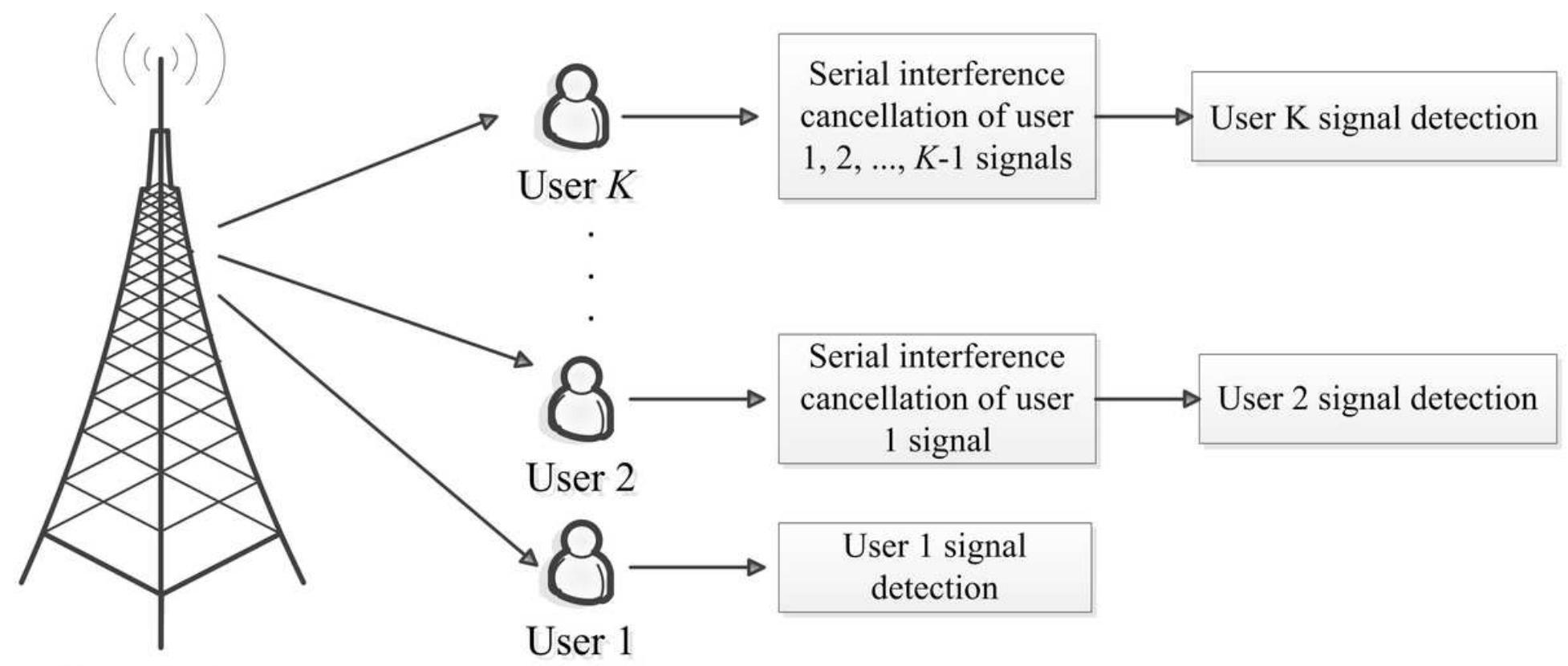

\section{Base Station}

Figure 1

User detection model 


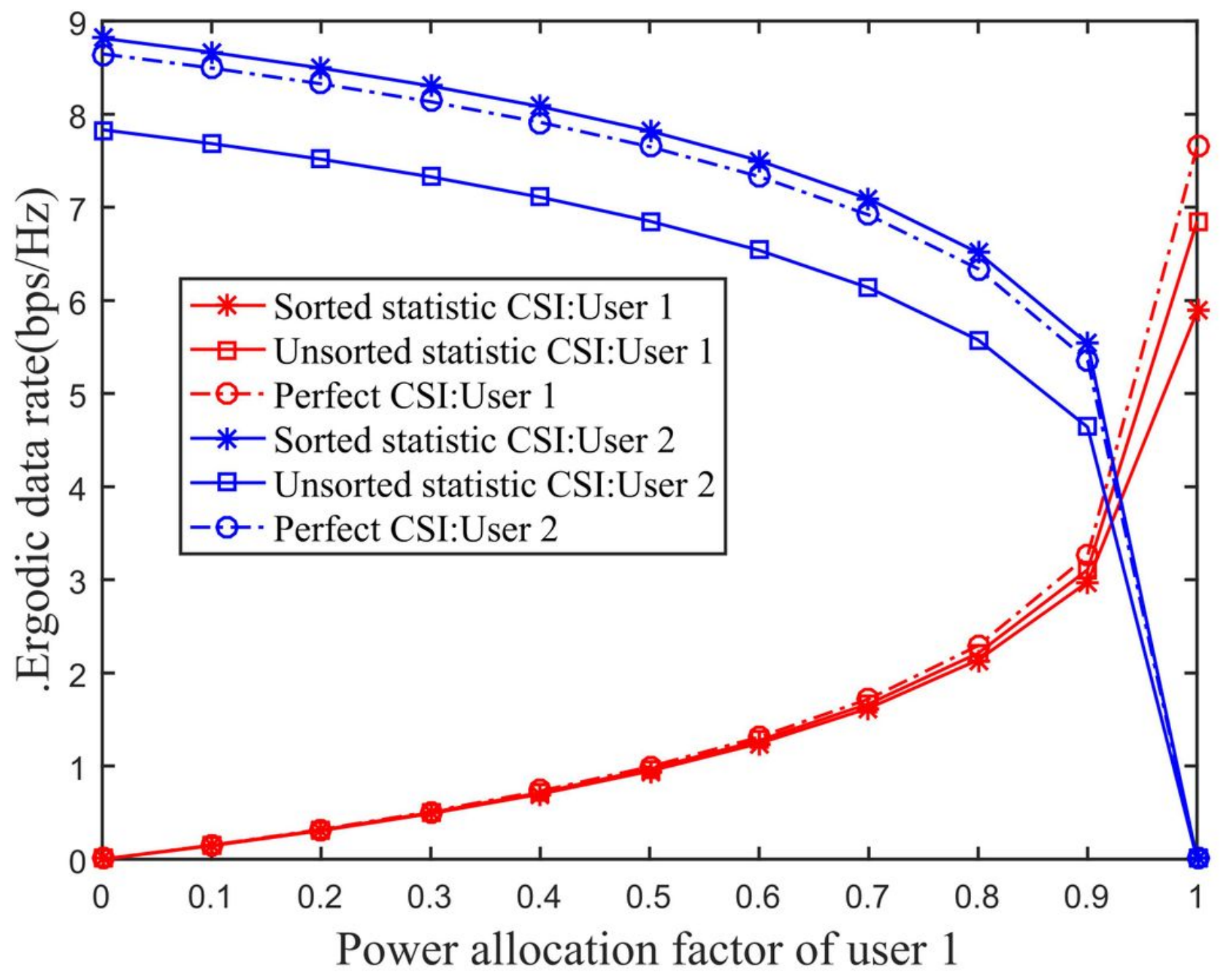

Figure 2

Comparison of ergodic date rate of NOMA users with statistical CSI and perfect CSI 


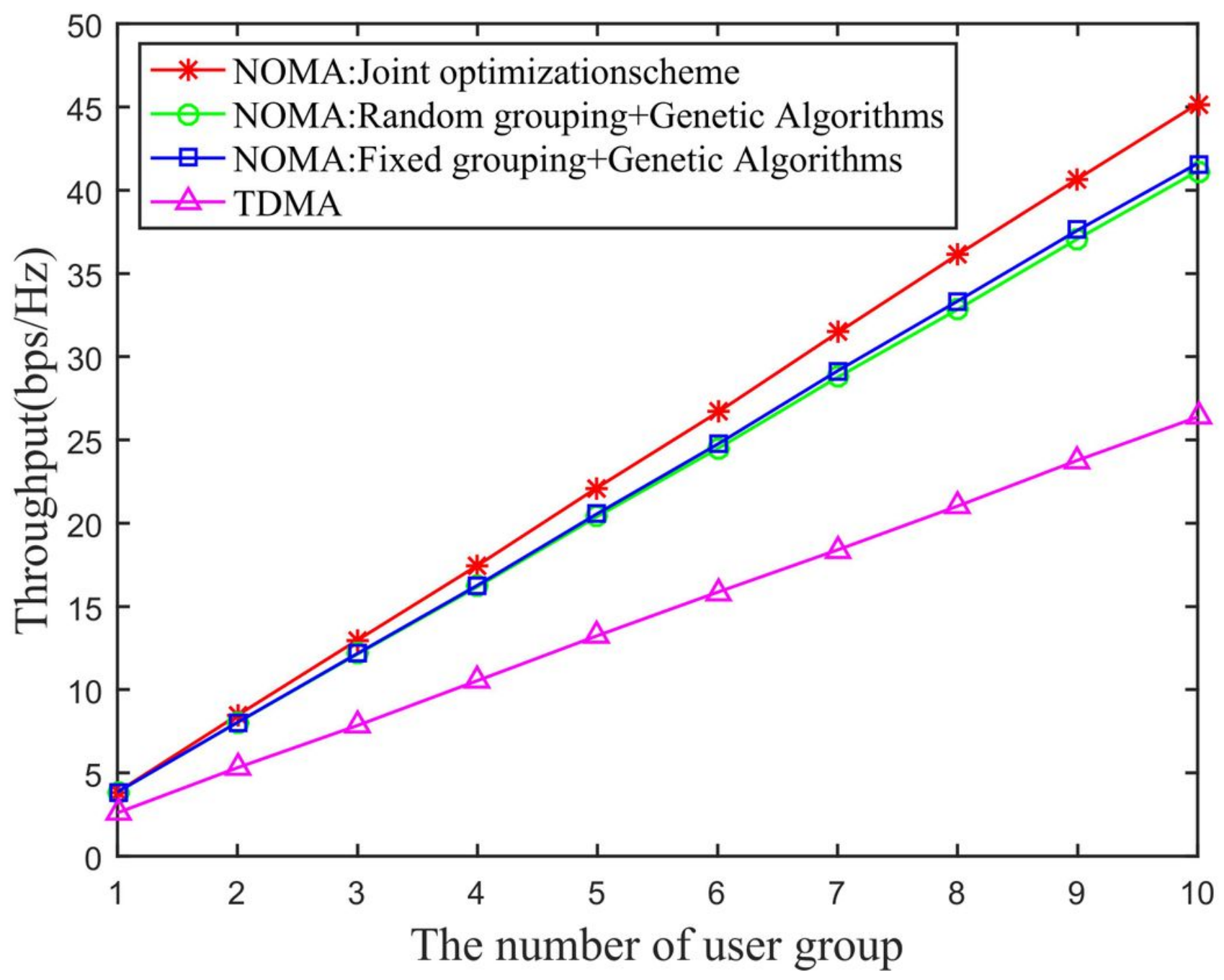

Figure 3

Trend of system throughput with the number of users 


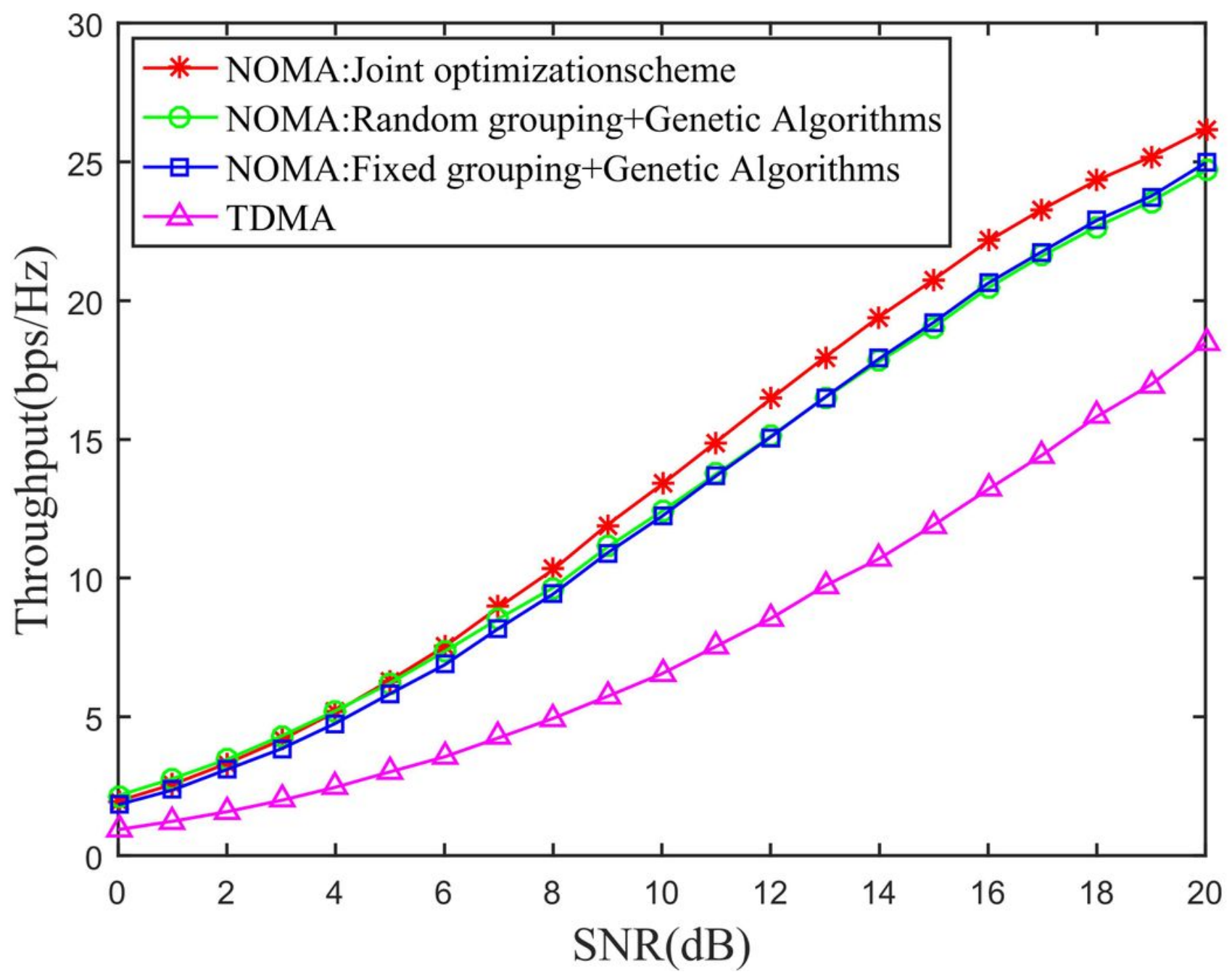

Figure 4

Trend of system throughput with the SINR 